\title{
Interaction of $\mathbf{C}_{60}$ with Carbon Nanotubes and Graphite
}

\author{
Hendrik Ulbricht, Gunnar Moos, and Tobias Hertel \\ Fritz-Haber-Institut der Max-Planck-Gesellschaft, Faradayweg 4-6, 14195 Berlin, Germany
}

(Received 6 August 2002; published 3 March 2003)

\begin{abstract}
The interaction of $\mathrm{C}_{60}$ with single-wall carbon nanotubes (SWNTs) and graphite is studied experimentally by thermal desorption spectroscopy and theoretically by molecular-mechanics and molecular-dynamics calculations. The van der Waals parameters and force field for $\mathrm{C}_{60}$-graphene and $\mathrm{C}_{60}$-SWNT interactions are derived from the low-coverage $\mathrm{C}_{60}$ binding energy to the graphite surface. We use these to compare the efficiency of different mechanisms by which $\mathrm{C}_{60}$ can be encapsulated into SWNTs.
\end{abstract}

DOI: 10.1103/PhysRevLett.90.095501

PACS numbers: 61.48.+c, 61.46.+w, 68.43.Vx, 83.10.Rs

Carbon nanotubes (CNTs) have unique potential as multifunctional materials in nanoscaled gas sensors [1], molecular field effect transistors [2,3], as additives for composite materials [4], and more. Their synthesis and functionalization, aiming at the fabrication of materials with tailored properties, present an important challenge in the exploitation of their potential for future technologies. Our understanding of the microscopic mechanisms and forces underlying the formation of functionalized CNTs and CNT-hybrid structures is an important prerequisite for the successful optimization of such fabrication processes.

The combination of one-dimensional single-wall carbon nanotubes (SWNTs) with zero-dimensional fullerenes by encapsulation of $\mathrm{C}_{60}$ into the nanotubes [5,6] yields so-called "peapods"- a material with unique electronic structure [7] whose properties are currently studied with great effort. In particular, the mechanism of $\mathrm{C}_{60}$ encapsulation is still discussed controversially [8]. The interaction of $\mathrm{C}_{60}$ with the graphitic surface of SWNTs is expected to be predominantly through dispersion forces which are also important for the cohesive energy of graphite and $\mathrm{C}_{60}$ crystals $[9,10]$. However, dispersion forces are notoriously difficult to determine theoretically, and it was noted early on that experimental data are needed as a benchmark for further theoretical investigations [11]. Here, we report on the first experimental determination of van der Waals (VDW) parameters describing $\mathrm{C}_{60}$-graphene and $\mathrm{C}_{60}$-CNT interactions. Previous VDW potentials for $\mathrm{C}_{60}-\mathrm{SWNT}$ interactions were based on the combination of $\mathrm{C}_{60}-\mathrm{C}_{60}$ VDW parameters with those obtained for graphene-graphene interactions using the empirical combining rules for VDW potentials $[12,13]$. The scarce experimental data on the strength of VDW interactions in graphitic systems in general and its complete nonexistence for the $\mathrm{C}_{60}$-graphene interaction, in particular, stimulated this investigation in an attempt to determine VDW parameters which permit one to explore the energetics and dynamics of $\mathrm{C}_{60}$-SWNT interactions in further detail. To this end, we studied the interaction of $\mathrm{C}_{60}$ with highly oriented pyrolytic graphite (HOPG) and SWNT bundles using thermal desorption spectroscopy. Empirical VDW constants are derived from the $\mathrm{C}_{60}$-graphite binding energy. This allows one to calculate $\mathrm{C}_{60}$-SWNT potential energy surfaces (PESs) and identify possible reaction paths on which $\mathrm{C}_{60}$ can be encapsulated into SWNTs.

SWNT samples are made from purified nanotube suspension (tubes@rice, Houston, Texas) [14]. HOPG and SWNT samples were cleaned in situ by repeated vacuum annealing to $1200 \mathrm{~K}$ under ultrahigh vacuum conditions. $\mathrm{C}_{60}$ of $99.5 \%$ purity is sublimed from a Knudsen cell at a rate of typically 0.1 monolayers (ML) per minute. Desorption of $\mathrm{C}_{60}$ was monitored using the total pressure in the UHV chamber as measured by an ion gauge. Background correction of thermal desorption (TD) traces was carefully cross-checked with TD spectra from species with masses between 4 and 200 a.m.u., the range of our quadrupole mass spectrometer. Absolute coverages and desorption rates were calibrated using the TD spectrum from a saturated and close-packed $\mathrm{C}_{60}$ monolayer on HOPG prepared by annealing a few ML thick $\mathrm{C}_{60}$ films. Saturation of the HOPG surface with $\mathrm{C}_{60}$ could be probed using Xe TD spectra which exhibit distinctly different features if $\mathrm{Xe}$ is desorbed from $\mathrm{C}_{60}$ or HOPG surfaces. The heating rate was $2.0 \mathrm{~K} \mathrm{~s}^{-1}$. Additional experimental details can be found elsewhere [15].

The low-coverage TD spectra from the HOPG surface in Fig. 1(a) are typical for zero order desorption kinetics. This is attributed to attractive adsorbate-adsorbate interactions frequently observed for gases physisorbed on graphite. Desorption from the second layer is evident from the additional desorption feature starting on the rising edge of the monolayer feature. The shape and evolution of spectra are consistent with desorption from three-dimensional islands grown by a Volmer-Weber-type mode as reported by a STM study [16]. A careful analysis of TD traces was performed using modified zero order kinetic rate equations where desorption from the $n$th layer is given by 


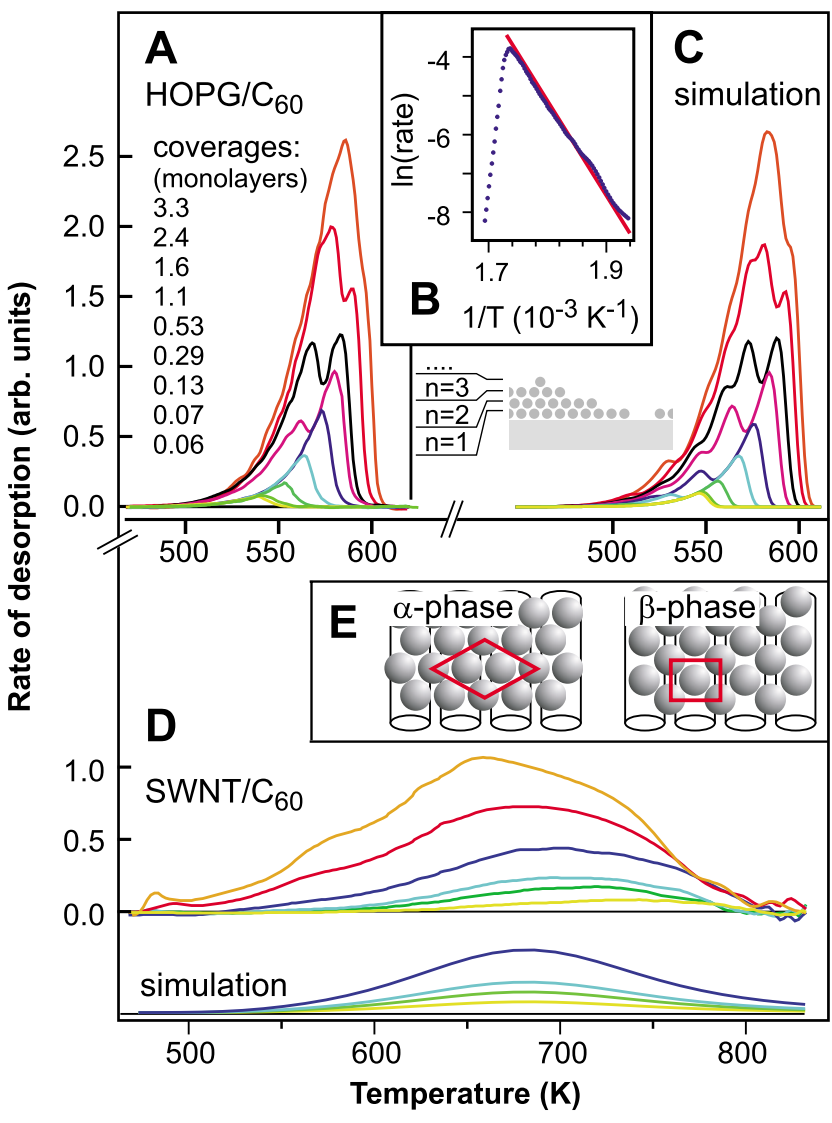

FIG. 1 (color). Thermal desorption spectra from HOPG and SWNT samples. (a) Series of TD spectra after adsorption of 0.06-3.3 ML of $\mathrm{C}_{60}$ on HOPG. (b) Arrhenius plot of the desorption rate at low coverage. (c) Calculated spectra using a multilayer desorption scenario. (d) Spectra from SWNT samples after exposure to similar amounts of $\mathrm{C}_{60}$ are broadened and shifted to higher temperatures as are the calculated traces indicating a small increase of the activation energy for desorption from SWNT bundles. (e) Conceivable adsorbate layer arrangements on an idealized SWNT substrate. $\alpha$ and $\beta$ phases exhibit the largest number of highly coordinated sites within an almost close-packed layer. Unit cells are marked by red lines.

$$
\frac{d \Theta_{n}}{d t}=-\nu_{n}\left(1-\Theta_{n+1}\right) \exp \left(-\frac{E_{B}}{k_{B} T}\right) .
$$

The factor $\left(1-\Theta_{n+1}\right)$ accounts for the constraint of the rate of desorption from the $n$th layer by the coverage $\Theta_{n+1}$ of the $(n+1)$ th layer. The preexponential factor $\nu$ for desorption from $\mathrm{C}_{60}$ multilayers, $10^{13.2 \pm 0.3} \mathrm{~s}^{-1}$, is obtained from the temperature dependence of the $\mathrm{C}_{60}$ vapor pressure [17]. Preexponentials for desorption of VDW bonded monolayers are generally very close to those for desorption from the corresponding solid such that we can use the same factor for desorption from all layers. Our analysis reveals that the activation energy for desorption from the first monolayer is only $(2 \pm 1) \%$ higher than for desorption from the multilayers. This yields an activation energy for $\mathrm{C}_{60}$ desorption from the first layer of $1.69 \mathrm{eV}$, for which the heat of sublimation $E_{\text {sub }}$ from the bulk $\mathrm{C}_{60}$ phase of $1.65 \mathrm{eV}$ [17] was used as a reference. Calculated TD traces are shown in Fig. 1(c) where initial coverages needed for the integration of Eq. (1) were estimated from the sequential saturation of individual layers expected for three-dimensional, Volmer-Weber-type growth. Identical results are obtained from the analysis of the rising edge in submonolayer TD spectra shown in Fig. 1(b) as well as from the temperature of the desorption maximum at saturation of the first monolayer.

The activation energies for desorption of VDW bonded adsorbates from graphite commonly include contributions from the binding energy to the surface and the binding energy within two-dimensional adsorbate islands in which the adsorbates are agglomerated. From the common leading edge of the low-coverage TD traces in Fig. 1(a) one can infer that these islands are stable up to at least $580 \mathrm{~K}$, the temperature of desorption. Consequently, the binding energy of a single $\mathrm{C}_{60}$ molecule to graphite, $0.85 \mathrm{eV}$, can be estimated by subtraction of the island binding energy - about $1 / 12$ the cohesive energy of solid $\mathrm{C}_{60}$ for each nearest neighbor, i.e., approximately $6 / 12 E_{\text {sub }}$.

These values now provide experimental benchmarks guiding the choice of VDW parameters for $\mathrm{C}_{60}$-graphene interactions. To convert the measured binding energy into the appropriate potential parameters, we use the familiar Lennard-Jones potential:

$$
V(r)=\frac{C_{12}}{r^{12}}-\frac{C_{6}}{r^{6}}
$$

We then calculate the dependence of different material properties on the $\mathrm{C}_{6}$ and $\mathrm{C}_{12}$ parameters by summation of VDW pair potentials for different structures; see Fig. 2. The binding energy of $\mathrm{C}_{60}$ to the graphite surface, including attractive interactions within two-dimensional $\mathrm{C}_{60}$ islands, requires that $\left(\mathrm{C}_{6}, \mathrm{C}_{12}\right)$ parameters are chosen from a point on the long-dashed line. Because of a lack of additional experimental reference data for the $\mathrm{C}_{60}$-graphite system we here chose VDW parameters to match the desorption energy and the graphite lattice constant (dot-dashed line in Fig. 2). The latter should ensure a reasonable description of the repulsive part of the interaction potential. The values used in the following are $\mathrm{C}_{12}=22500 \mathrm{eV} \AA^{12}$ and $\mathrm{C}_{6}=15.4 \mathrm{eV} \AA^{6}$. Within the range of reported values, these parameters also reproduce the graphite $c$-axis compressibility, the cohesive energy of graphene sheets in graphite, and the VDW contribution to the $\mathrm{C}_{60}$ bulk cohesive energy reasonably well. For comparison we have included the parameters used for different graphitic systems by Girifalco et al. [13] and for bulk $\mathrm{C}_{60}$ interactions by Lu et al. [10].

For SWNTs, the curvature of graphene sheets and the resulting asymmetry of $\pi$ orbitals may induce changes to binding energies on interior and exterior SWNT surfaces. For tube diameters used here we expect such corrections 

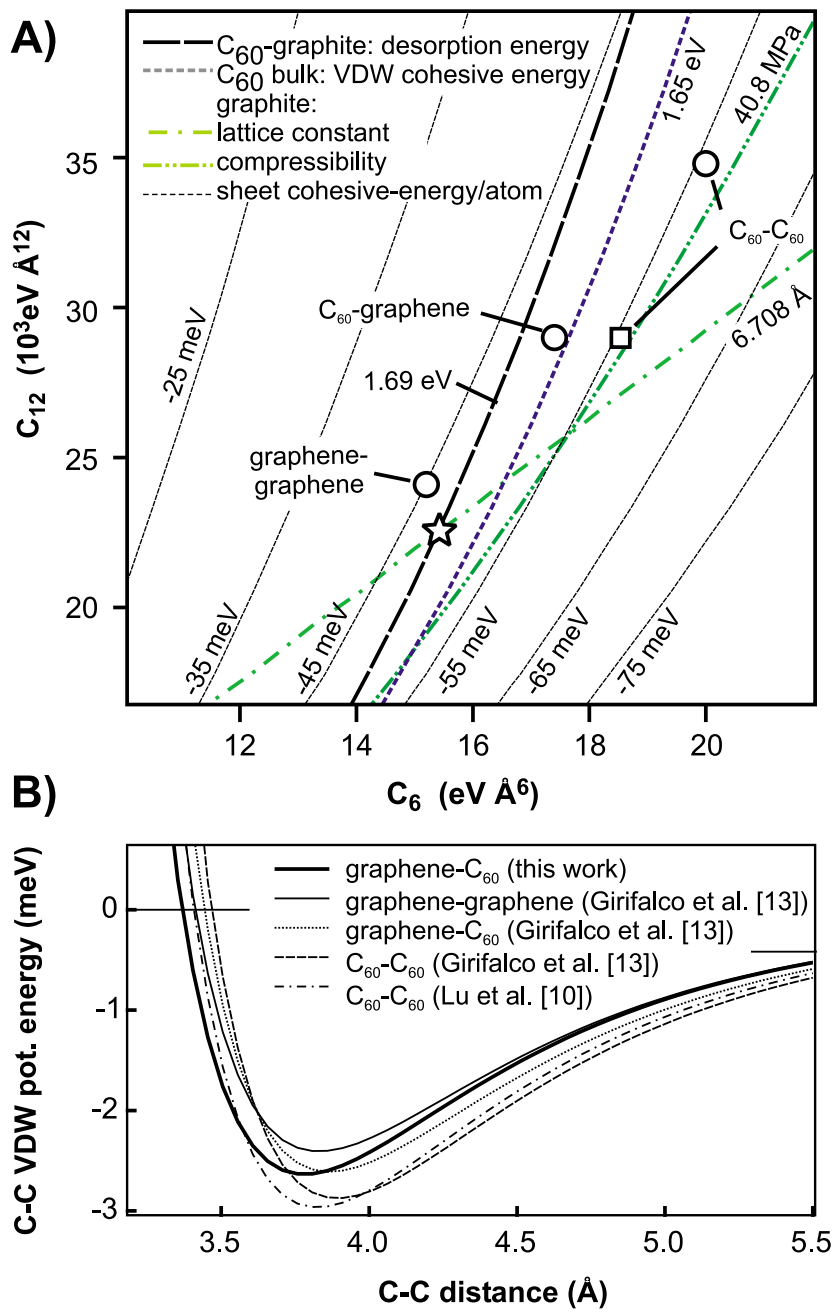

FIG. 2 (color online). (a) van der Waals parameters and the resulting material properties for different systems. $\left(\mathrm{C}_{6}, \mathrm{C}_{12}\right.$ parameters on the long-dashed line, for example, yield the measured activation energy for $\mathrm{C}_{60}$ desorption from graphite. To simultaneously account for properties of graphite such as lattice constant or $c$-axis compressibility we chose a set of $\left(\mathrm{C}_{6}, \mathrm{C}_{12}\right)$ parameters in the lower part of the graph, marked by a star. The VDW parameters used by Lu et al. [10] and Girifalco et al. $[12,13]$ are given by open squares and circles, respectively. (b) The corresponding Lennard-Jones potentials.

to scale with the misalignment of $\pi$ orbitals which-if similar to curvature corrections to the electronic structure - would be of the order of $1 \%-2 \%$ [18]. This does not lead to any significant change of the dynamics or encapsulation probabilities along different reaction paths, and curvature effects are thus neglected in the following discussion.

In the case of thermal $\mathrm{C}_{60}$ desorption from SWNT samples, the best agreement of calculated with experimental TD traces is obtained for a desorption energy of $1.7 \pm 0.2 \mathrm{eV}$ using a kinetic model introduced elsewhere [15]. The above VDW potential can qualitatively account for this if one assumes that $\mathrm{C}_{60}$ is arranged on the SWNT bundle surface in a close-packed manner. Two energeti-

095501-3 cally favored configurations ( $\alpha$ and $\beta$ phases) in which $\mathrm{C}_{60}$ molecules would be mostly adsorbed in highly coordinated sites on the external bundle surface are illustrated in Fig. 1(e). They are determined by the small mismatch of the $(9,9)$ SWNT bundle lattice constant, $15.3 \AA$ with $3 / 2$ the $\mathrm{C}_{60}$ bulk spacing, $15.03 \AA$ and with the $\mathrm{C}_{60}$ bulk fcc lattice constant of $14.17 \AA$. Using the above potential we calculate the $\mathrm{C}_{60}$ binding energy in the so-called "groove sites" to be $1.02 \mathrm{eV}$ while it should be about $0.54 \mathrm{eV}$ elsewhere. Including 6/12 $E_{\text {sub }}$ and 4/12 $E_{\text {sub }}$ for the intralayer binding energies - for six and four nearest neighbors - this would yield $\mathrm{C}_{60}$ desorption energies of $1.5 \mathrm{eV}$ and $1.3 \mathrm{eV}$ for the tentatively proposed $\alpha$ and $\beta$ phases, respectively.

This qualitative agreement with experimental observations encourages us to use the above potentials to discuss the mechanism by which $\mathrm{C}_{60}$ is encapsulated in SWNTs. Generally, two distinct mechanisms are conceivable: (a) direct "ballistic" encapsulation of $\mathrm{C}_{60}$ from the gas phase through open ends or defects in the tube walls or (b) migration of adsorbed $\mathrm{C}_{60}$ from the external tube and bundle surfaces into the SWNT interior [8]. To illustrate the different reaction paths studied here we have reproduced a triple- $(10,10)$ tube arrangement in Fig. 3 which has all the major structural features to be expected in real systems: (1) isolated open ends, (2) nonisolated open ends, and (3) defect openings. As shown in the lower part of Fig. 3 the potential along the reaction path into the individual tube end is found to increase from -0.54 to
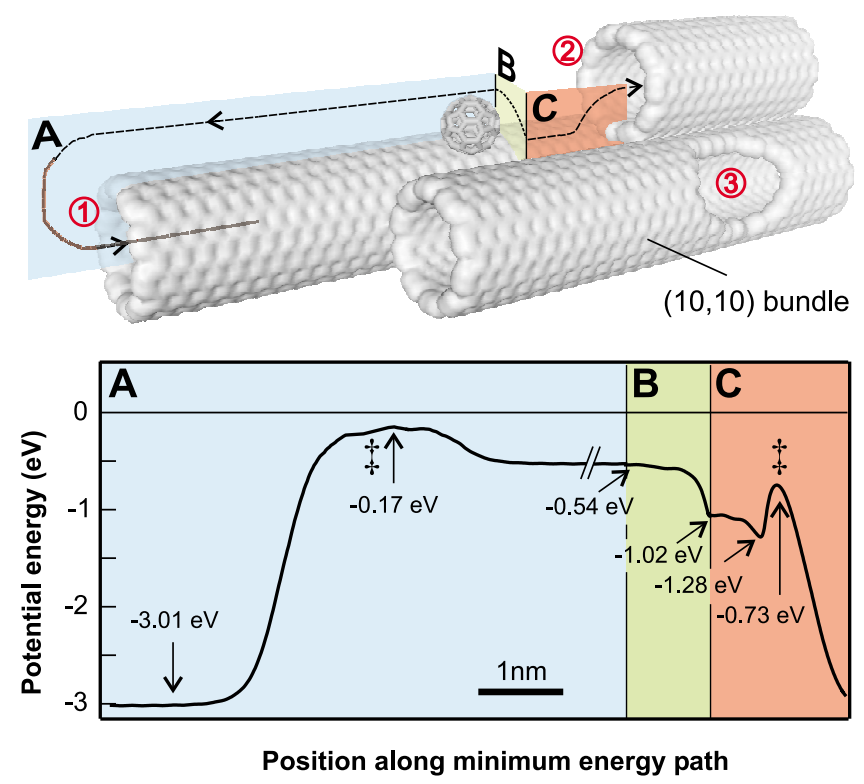

FIG. 3 (color online). Cut through three-dimensional potential energy surface of a small $(10,10)$ tube bundle. Spontaneous encapsulation along all relevant reactions paths is prevented by lip barriers (LBs) due to the lower $\mathrm{C}_{60}$ coordination at the rim of SWNTs. Calculations show that encapsulation is most efficient via the head-on approach along the reaction path labeled (c).Transition states are marked by a double dagger $(\$)$.

095501-3 
about $-0.17 \mathrm{eV}$ before it drops dramatically to $-3.01 \mathrm{eV}$ when $\mathrm{C}_{60}$ moves along the outside of the tube over the lip edge into the tube. Similar endohedral binding energies have also been obtained by Girifaclo et al. $[12,13]$. The large effective barrier for overcoming the lip edge of $0.37 \mathrm{eV}$ is here due to the reduced coordination of $\mathrm{C}_{60}$ at the tube end and should prevent $\mathrm{C}_{60}$ from spontaneous encapsulation. The origin of this LB is attributed to reduced coordination in analogy to the familiar EhrlichSchwoebel barriers near step edges of solid surfaces [19]. Similar barriers are found for all possible surface migration paths into the open tubes.

To identify which reaction path eventually facilitates most efficiently encapsulation we have performed threedimensional kinetic Monte Carlo trajectory calculations on a rigid bundle similar to the one shown in Fig. 3. Twodimensional calculations on cuts through the 3D PES would yield misleading results owing to the importance of angular momentum for the encapsulation process. Trajectories were propagated by the velocity Verlet algorithm such that typically $10^{5}$ had energies above the encapsulation threshold for each tested path. The temperature of the statistical ensemble, $650^{\circ} \mathrm{C}$, is close to that of experimental encapsulation runs [6]. Trajectories were defined as successful if their approach from the entrance channel of the PES leads to encapsulation after passing the transition state in less than 100 ps. The probability for encapsulation via the groove at the bottom of a tube opening into the open end, $5 \times 10^{-3}$, was found to be highest, while for encapsulation through defect openings or over the isolated LB it was found to be $6 \times$ $10^{-5}$ and $3 \times 10^{-5}$, respectively. Other conceivable paths were less probable. For comparison with experiments the encapsulation probability needs to be weighted by the attempt frequency resulting from the concentration in a particular entrance channel. Surface concentrations were estimated using the above binding energies and assuming Langmuir kinetics at equilibrium with the $\mathrm{C}_{60}$ vapor phase, $650{ }^{\circ} \mathrm{C}$ and $10 \mathrm{~Pa}$. For the groove as entrance channel this yields a concentration of $5 \times 10^{5} \mathrm{~m}^{-1}$ resulting in an attempt frequency of about $2 \times 10^{7} \mathrm{~s}^{-1}$. The attempt frequency for entry over the lip of a freestanding tube end is only about $2 \times 10^{4} \mathrm{~s}^{-1}$ due to the small $\mathrm{C}_{60}$ concentration on these low-coordinated, i.e., less strongly binding, sections of the bundle. This eventually leads to a clear preference of the direct "head-on" encapsulation path (c). The rate of successful encapsulation attempts for the defect openings and isolated tube ends are estimated to be $10^{3}$ and $10^{5}$ times smaller, respectively. The rate of ballistic capture from the gas phase through the $2 \AA^{2}$ entrance of an open $(10,10)$ tube end would likewise be about 2 orders of magnitude smaller than the head-on capture mechanism.

A direct comparison of the resulting rates with experimental findings is challenging as global filling kinetics are expected to depend on a number of additional factors such as the distribution of tube diameters in the mixed samples and the accessibility of openings. Despite the lack of detailed kinetic data, the results presented here suggest that the calculated filling rates by far exceed what experiments indicate. While some authors have reported filling ratios of up to $70 \%$ within less than $2 \mathrm{~h}$ at $650^{\circ} \mathrm{C}$ [6], others report on an increase in the overall filling ratio over several days [20]. In contrast, the above calculations suggest that tubes could, in principle, be filled within seconds.

In conclusion, we have found that $\mathrm{C}_{60}$ encapsulation by SWNTs should proceed most rapidly via migration of adsorbed fullerenes and not by ballistic capture from the gas phase despite the significant Ehrlich-Schwobellike barriers near all types of tube openings. Encapsulation should be most probable for $\mathrm{C}_{60}$ adsorbed in grooves by head-on collisions with open tube ends. The high predicted rate of successful encapsulation attempts moreover suggests that the experimentally observed filling rates are not limited by the probability that $\mathrm{C}_{60}$ molecules find their way to the entrance of an open tube but rather by the rate at which encapsulated $\mathrm{C}_{60}$ molecules or chains of molecules migrate inside the tubes. Interaction with defects on the tube walls or the pressure from residual gas trapped inside of tubes may make it difficult for $\mathrm{C}_{60}$ chains to freely migrate and thus slow down the encapsulation process despite the large driving forces expected at the tube entrance.

We thank Gerhard Ertl for his continuing and generous support of this work.

[1] J. Kong et al., Science 287, 622 (2000).

[2] S. J. Tans et al., Nature (London) 393, 49 (1998).

[3] R. Martel et al., Appl. Phys. Lett. 73, 247 (1998).

[4] B. Vigolo et al., Science 290, 1331 (2002).

[5] B.W. Smith et al., Nature (London) 396, 323 (1998).

[6] H. Kataura et al., Synth. Met. 121, 1195 (2001).

[7] D. J. Hornbaker et al., Science 295, 828 (2002).

[8] S. Berber et al., Phys. Rev. Lett. 88, 185502 (2002).

[9] S. Saito and A. Oshiyama, Phys. Rev. Lett. 66, 2637 (1991).

[10] J. P. Lu et al., Phys. Rev. Lett. 68, 1551 (1992); X.-P. Li et al., Phys. Rev. B 46, 4301 (1992).

[11] Ch. Girard et al., Phys. Rev. B 49, 11425 (1994).

[12] L. A. Girifalco and R. A. Lad, J. Chem. Phys. 25, 693 (1956).

[13] L. A. Girifalco et al., Phys. Rev. B 62, 13104 (2000).

[14] A. G. Rinzler et al., Appl. Phys. A 67, 29 (1998).

[15] H. Ulbricht et al., Chem. Phys. Lett. 363, 252 (2002).

[16] H. Yu et al., Surf. Sci. 286, 116 (1993).

[17] J. Abrefah et al., Appl. Phys. Lett. 60, 1313 (1992).

[18] J.W. Ding et al., Phys. Rev. B 66, 073401 (2002).

[19] G. Ehrlicha and F. G. Hudda, J. Chem. Phys. 44, 1039 (1966); R. L. Schwoebel and E. J. Shipsey, J. Appl. Phys. 37, 3682 (1966).

[20] B.W. Smith et al., J. Appl. Phys. 91, 9333 (2002). 